\title{
DDESC: Dragon database for exploration of sodium channels in
} human

\author{
Sunil Sagar, Mandeep Kaur, Adam Dawe, \\ Sundararajan Vijayaraghava Seshadri, Alan Christoffels, Ulf Schaefer, \\ Aleksandar Radovanovic and Vladimir B Bajic*
}

\begin{abstract}
Address: South African National Bioinformatics Institute, University of the Western Cape, Private Bag- X17, Modderdam Road, Bellville, Cape Town 7535, South Africa

Email: Sunil Sagar - sunil@sanbi.ac.za; Mandeep Kaur - mandeep@sanbi.ac.za; Adam Dawe - adam@sanbi.ac.za;

Sundararajan Vijayaraghava Seshadri - sundar@sanbi.ac.za; Alan Christoffels - alan@sanbi.ac.za; Ulf Schaefer - ulf@sanbi.ac.za;

Aleksandar Radovanovic - alex@sanbi.ac.za; Vladimir B Bajic* - vlad@sanbi.ac.za

* Corresponding author
\end{abstract}

Published: 20 December 2008

BMC Genomics 2008, 9:622 doi:10.1/86/147|-2164-9-622
Received: 27 October 2008

Accepted: 20 December 2008

This article is available from: http://www.biomedcentral.com/l47/-2/64/9/622

(c) 2008 Sagar et al; licensee BioMed Central Ltd.

This is an Open Access article distributed under the terms of the Creative Commons Attribution License (http://creativecommons.org/licenses/by/2.0), which permits unrestricted use, distribution, and reproduction in any medium, provided the original work is properly cited.

\begin{abstract}
Background: Sodium channels are heteromultimeric, integral membrane proteins that belong to a superfamily of ion channels. The mutations in genes encoding for sodium channel proteins have been linked with several inherited genetic disorders such as febrile epilepsy, Brugada syndrome, ventricular fibrillation, long QT syndrome, or channelopathy associated insensitivity to pain. In spite of these significant effects that sodium channel proteins/genes could have on human health, there is no publicly available resource focused on sodium channels that would support exploration of the sodium channel related information.

Results: We report here Dragon Database for Exploration of Sodium Channels in Human (DDESC), which provides comprehensive information related to sodium channels regarding different entities, such as "genes and proteins", "metabolites and enzymes", "toxins", "chemicals with pharmacological effects", "disease concepts", "human anatomy", "pathways and pathway reactions" and their potential links. DDESC is compiled based on text- and data-mining. It allows users to explore potential associations between different entities related to sodium channels in human, as well as to automatically generate novel hypotheses.

Conclusion: DDESC is first publicly available resource where the information related to sodium channels in human can be explored at different levels. This database is freely accessible for academic and non-profit users via the worldwide web http://apps.sanbi.ac.za/ddesc.
\end{abstract}

\section{Background}

Sodium channels are heteromultimeric, integral membrane proteins that conduct the sodium ions $\left(\mathrm{Na}^{+}\right)$ through plasma membrane of the cell. The classification of sodium channels is based on the trigger that opens the channel for ions, i.e. voltage-gated sodium channels (triggered by a voltage-change) and ligand-gated sodium channels (triggered by binding of a ligand to the channel) [1]. The mutations in genes coding for sodium channel proteins have been linked with several genetic disorders, 
called 'sodium channelopathies' such as inherited febrile epilepsy, autism, Brugada syndrome, ventricular fibrillation, long QT syndrome, etc [2-7]. Recently, SCN9A gene which encodes for $\mathrm{NaV1.7}$ voltage-gated sodium channel, has been linked to molecular pathophysiologies of pain disorders like inherited erythromelalgia and inherited paroxysmal extreme pain disorder (PEPD) and has emerged as a therapeutic target for treatment of neuropathic pain [8] Additionally, nearly 20 disorders affecting skeletal muscle contraction, cardiac rhythm, or neuronal function have been linked to these mutations in human. It has also been shown that sodium channel mutations could cause alteration in the physiological properties (hyperexcitability or hypoexcitability) of the cells depending upon which sodium channels genes are expressed. Both sodium channel mutations and cell background contribute to neuronal function and clinical manifestations [9].

Due to the complexity of molecular functioning and effects that sodium channels have, it is important for biologists and medical researchers to have means to explore the relevant information in an easy fashion. However, the information regarding the sodium channels is scattered through the literature or across various public and commercial databases. To the best of our knowledge there is no resource focused specifically to sodium channels, though there are two publicly accessible ion channel databases: the Ion Channel Database http://www.ionchan nels.org/database.php and International Union of Pharmacology database http://www.iuphar-db.org/[10]. These databases provide mainly sequence information about the genes encoding for different ion channels. Consequently, there is a need for a focused comprehensive public resource that allows users to explore information related to sodium channels from multiple angles.

As the amount of scientific literature continues to increase, text-mining is becoming more important in extracting and summarizing information from the literature. Text-mining also ensures the investigation of latest and wider range of publications. We present here Dragon Database for Exploration of Sodium Channels in Human (DDESC), a sodium channel biology resource, compiled based on text- and data-mining. It provides comprehensive information about genes and proteins, metabolites and enzymes, toxins, chemicals with pharmacological effect, disease concepts, human anatomy, pathways and pathway reactions, potentially associated with sodium channel, and provides potential links between these entities. The present study introduces a database for exploring human sodium channels in order to provide useful information for drug development. Various computational approaches, such as structural bioinformatics [11-14], molecular docking [15-20], pharmacophore modelling
[21], QSAR [22-27], protein sub-cellular location prediction $[28,29]$, identification of membrane proteins and their types [30], identification of enzymes and their functional classes [31], identification of proteases and their types [32], protein cleavage site prediction [33-35], and signal peptide prediction [36,37], provide useful information and insights for both basic research and drug design. All these fields of research can further benefit from DDESC and hence the database can serve a wider science community. The database is accessible via the worldwide web http://apps.sanbi.ac.za/ddesc, where it will be regularly updated. The access is free for academic and nonprofit users.

\section{Construction}

DDESC is generated by the licensed OrionCell's http:// www.orioncell.org Dragon Exploration System (DES) tool. DES uses dictionary based text-mining approach for extracting potentially relevant information from text documents. The functioning of the text-mining modules of DDESC is based on similar concepts as used in [38] and [39]. DES has previously been utilized in the creation of a part of the DDOC database [40]. In our study, DES is applied with six OrionCell's proprietary and manually curated dictionaries for "human genes and proteins", "metabolites and enzymes", "toxins", "chemical with pharmacological effects", "disease concepts" and "human anatomy". The dictionaries contain numerous variants of names and symbols customary for the specific types of entities. For example the dictionary of genes and proteins contains over 300,000 variants of entities covering the names, symbols, aliases, previous names and previously used symbols of genes and proteins, compiled from the literature and published databases.

The information in DDESC was based on 5,243 abstracts retrieved from PubMed repository http:// www.ncbi.nlm.nih.gov on 2008-08-06 by using the query ("sodium channel" OR "sodium channels") human. DES then maps all the entities from the dictionaries to the documents submitted for the analysis and the extracted information is then compiled into a database. DDESC provides the summarized lists of entities, frequency of published documents, frequency of pairs of entities, as well as clustering of documents based on entities found. The details on how to use the database and other relevant details about the methods applied by DES are provided in the documentation http://apps.sanbi.ac.za/ddesc/ddesc.pdf.

\section{Utility}

To date, there is no resource available, which could provide detailed information about the various potential biological interactions related to sodium channels. The DDESC is the first publicly available resource where the user can explore multiple information regarding sodium 
channels at molecular, chemical and functional level (Fig 1). The information can be further curated and analysed in a constructive and systematic way. The database provides a user friendly interface in an easy to follow colorcoding and graphical representations.

All the entities in DDESC are further linked to the literature through the PubMed IDs. Each entity is also linked to Reactome pathways, as well as associated chemical reactions within the pathways. Another useful aspect of DDESC is its ability to generate association hypotheses for further investigation. User can generate the hypotheses by selecting any combination of the used dictionaries (see documentation). Associations between the different entities can also be viewed as a network. DDESC also provides search options by using simple logical operators i.e. "AND", "OR" and "NOT" that will further allow the users for easier and more direct access to each of the reports.

\section{Discussion}

DDESC is the first text- and data-mining based integrated knowledgebase that allows researchers to get an overview and explore efficiently the biology of sodium channels. For text-mining, a dictionary based method is used where comprehensive list of genes and other entities are matched against the documents for entity recognition and information extraction. If an entity is found, it is tagged as originating from the dictionary that is being processed at that moment. Due to the sequential processing of the dictionaries, it is not possible to tag already tagged entity again, even if such an entity exists in the other, not yet processed, dictionaries. Once all dictionaries are processed, all analysed PubMed abstracts are annotated by the entities from dictionaries as identified in the text. However the main difficulty in entity recognition is the lack of standardisation of the name. Each gene and protein has several synonyms and abbreviations, some of which are common English words. Entity association is based on the co-occurrence of the entities within the abstracts or sentences. If the two entities are repeatedly mentioned together, there are chances that they are linked directly or indirectly. The users could further explore such associations in the context of sodium channels by, for example, inspecting the documents from which the association was extracted. DDESC gives the user flexibility to change the reference dictionary to explore different type of entity associations (see documentation for details). Networks can be generated for the different types of entity associations, which can further be zoomed into the chosen terms and relations by expanding the correlation tree and selecting the preferred subsets of dictionaries. Networks help in bringing different types of data at one platform for its better understanding.

Hypothesis generation is another useful feature, which allows users to infer new relationships for different entities (Fig 2). The idea for the hypothesis generation is that if entity A is linked to entity B and entity B is further linked to entity $C$ then there may be a new relationship between entity A and entity C. Manually, it is very difficult to infer new hypothesis based on all the published facts, specially with the vast amount of available literature. Clicking on the 'test' link, one can further inspect validity of the hypothesis generated in DDESC, by retrieving the PubMed document(s) related to the entities linked through the hypothesis. If no such PubMed document is found, this could suggest a possible genuine new hypothesis for further exploration.

Evaluation of the accuracy of integrated data is a necessary step and generally is expressed in terms of precision (the ability to identify the correct entities in the abstracts relative to the number of all identified entities of the same type), recall (the ability to identify correct entities of specific type present in the abstracts relative to all such entities present in the abstracts) and F-measure [41]. Since it is not possible to evaluate every concept from each of the dictionaries across all 5,243 documents, we selected the SCN1A gene as the reference. The SCN1A gene is one of the most studied sodium channel genes and more than

\title{
FLUROTHYL $\rightarrow$
}

\begin{abstract}
NAIV)1.6 [1] SCN1A [1] SCN2A [1] SCN3A [1] SCNBA [1] SNEI [1] SODIUM [1]

[draw network | show hypotheses]

\section{Figure I}

Potential association of 'FLUROTHYL' a 'Chemical with pharmacological effects' with other entities. Clicking on the numbers next to the entities will open the relevant abstract(s). 


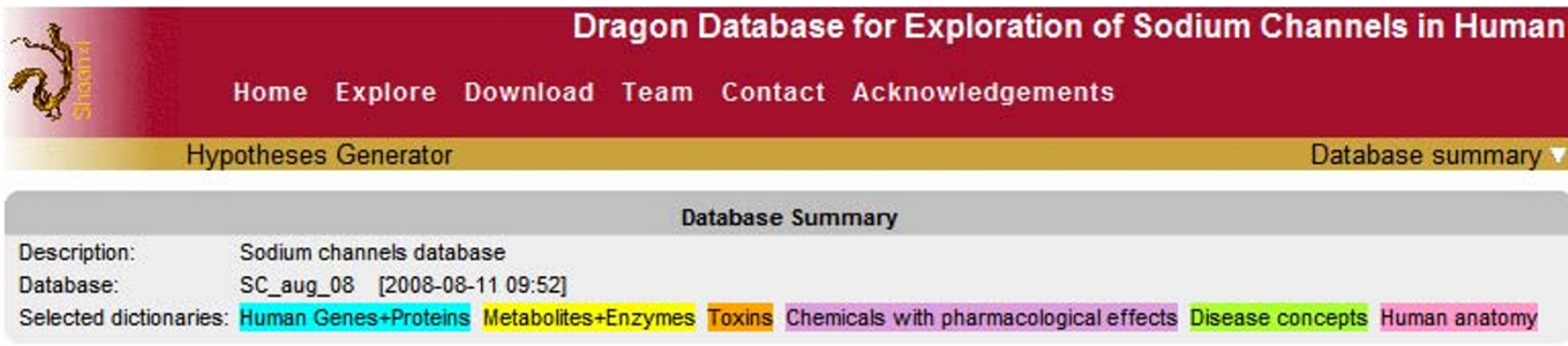

Page: $123456 \ldots 16$

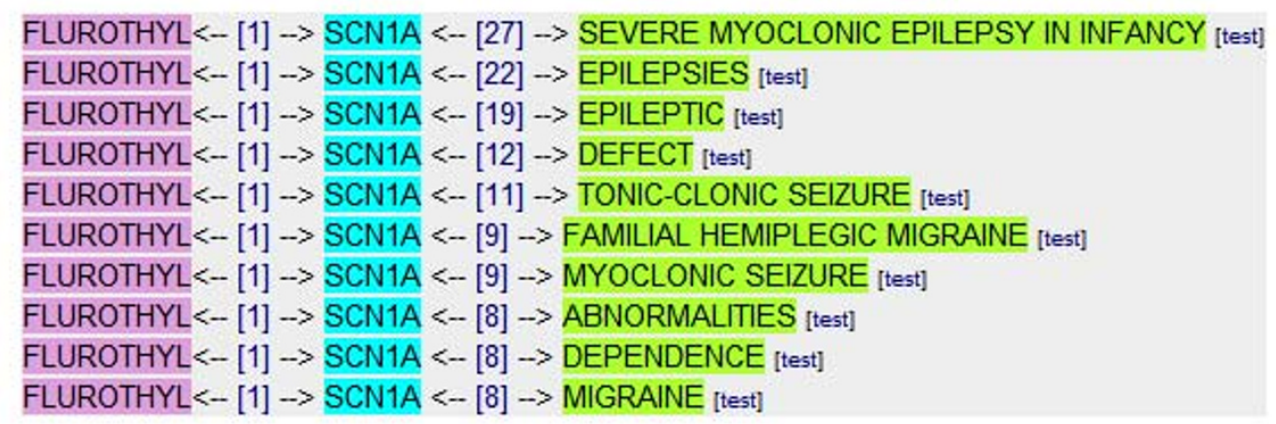

Page: $123456 \ldots 16$

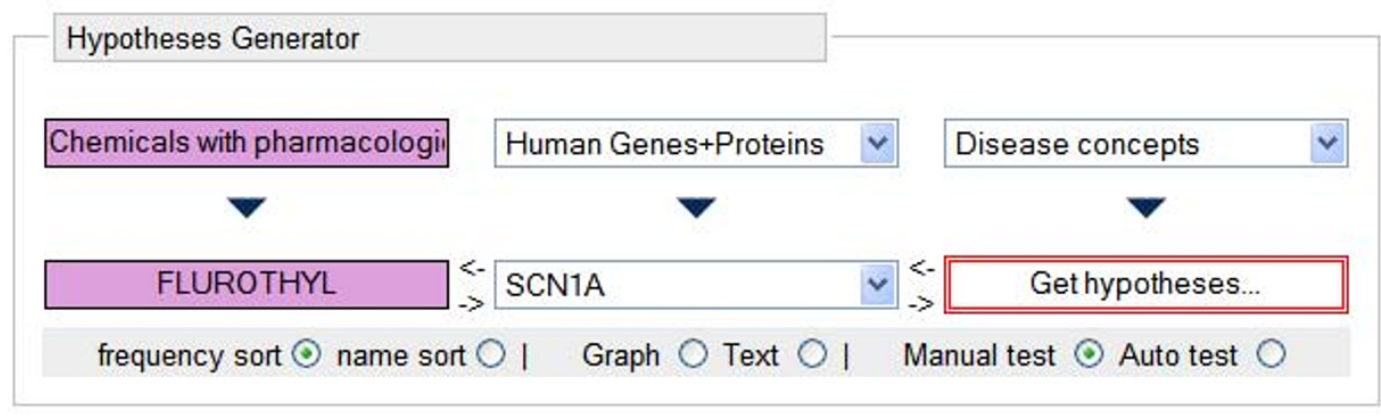

South African National Bioinformatics Institute \& OrionCell (9) 2008

\section{Figure 2}

Example of Hypotheses generated by using 'FLUROTHYL' under the dictionary 'Chemicals with pharmacological effects' and 'SCNIA' gene under the dictionary 'Human Genes and proteins'. At the time of writing this publication the 'test' link between 'FLUROTHYL' and 'EPILEPSIES' retrieved eight PubMed documents.

100 mutations have been identified within over $100 \mathrm{~kb}$ of its exon harbouring regions. SCN1A is also clinically very relevant as it contributes to the largest number of mutations linked with epilepsy [42]. For this purpose, we manually curated all 131 abstracts in which SCN1A gene has been explicitly identified. List of abstracts can be easily obtained on page 'summary of links'.

The analysis of the results for SCN1A gene shows that the precision and recall are in the range of $81 \%-100 \%$ (Table 1 ) depending on the type of dictionary used. Overall,
DDESC exploration database system has efficiently identified most of the entities from the 131 abstracts related to the SCN1A gene with an average F-measure value of $92.9 \%$. One should note, however, that these estimated quality parameters are only for the SCN1A gene and are based on 131 abstracts. The results may vary for the other entities. It is shown in Table 1 that $81.1 \%$ of the entities related to genes and proteins are correctly identified while 14 entities (SCN, SCN1, Potassium channel, VOLTAGEGATED K+ CHANNEL, VOLTAGE-GATED POTASSIUM CHANNEL, GTP, Parvalbumin, P17, P21, PL-3, AED, GEF 
Table I: Precision, recall and F-measure of entity recognition in documents related to SCNIA gene

\begin{tabular}{lllllr}
\hline Dictionaries used & Number of entities identified & $\begin{array}{l}\text { Number of correctly identified } \\
\text { entities }\end{array}$ & Precision (\%) & Recall (\%) & F-measure (\%) \\
\hline Genes and proteins & 74 & 60 & 81.1 & 96.1 & 87.9 \\
\hline Metabolites and enzymes & 28 & 28 & 100.0 & 100.0 & 100.0 \\
\hline $\begin{array}{l}\text { Chemicals with pharmacological } \\
\text { effects }\end{array}$ & 7 & 6 & 85.7 & 100.0 & 92.3 \\
\hline
\end{tabular}

and SMEI) were wrongly identified as genes or proteins. This is due to the fact that some of these entities refer to the family of genes (for example VOLTAGE-GATED POTASSIUM CHANNEL, SCN, CYP), some entities are recognized partly (for example, $S C N 1$ which is a part of SCN1-3A) and others are the synonyms of genes and proteins that are used as abbreviations for other biological entities in the abstracts. For example, SMEI is one of the aliases for SCN1A gene (sodium channel, voltage-gated, type I, alpha) and has been placed in the list of genes and proteins. However, in the relevant text SMEI actually referred to the term 'Severe myoclonic epilepsy of infancy' (PubMed ID: 11359211). Similarly GEF is a synonym for ARHGEF2 gene (rho/rac guanine nucleotide exchange factor (GEF) 2), but it is also an acronym for generalized epilepsy with febrile seizures plus (GEFS+).

In DDESC, more than $96 \%$ of genes and proteins symbols mentioned in 131 SCN1A-related abstracts were identified. The exceptions are $F H M 1, N a(v) 1.7$ and $N a(V) 1.1$ and these are synonyms for CACNA1A, SCN9A and $S C N 1 A$ genes respectively. In the list of identified chemicals with pharmacological effects, the only wrong term is 'lead', which is a metal and metabolite, but also a very common English word. Such entities contribute to lowering the precision. This is one of issues in the DES tool, which will require further improvements.

To check the efficiency of the system in comparison to others, we further did a brief comparison with recently published tool, PolySearch [43] The reported PolySearch F-measure for gene synonym identification, protein-protein interaction identification and disease gene identification is $88 \%, 81 \%$ and $79 \%$, respectively. We submitted the 'SCN1A' gene as a query to PolySearch and searched for metabolites and drugs related to SCN1A gene mentioned in these abstracts. PolySearch identified only six entities in total whereas DDESC has provided 28 metabolites and enzymes including drugs for SCN1A gene. The comparison in the category of genes and proteins shows that DDESC identified 31 more entities as compared to PolySearch, which identified 14 of them. This difference could be partially due to the content of the dictionaries.
For example, PolySearch has total approximately 234,000 entities for genes and proteins and their synonyms in their dictionary whereas DES contains over 300,000 entities for genes and proteins and their synonyms and other names. Detailed comparison of genes and proteins results can be found in Additional file 1.

We also looked if the entities identified actually do relate to sodium channels. Through manual curation of 131 abstracts, we identified entities directly associated with the functionality of sodium channels [see Additional file $1]$. In the category of "genes and proteins", 18 (30\%) out of total 60 entities were found to be either genes coding for various sodium channel proteins, or genes or proteins that could directly affect the functionality of sodium channel proteins. In the list of metabolites and enzymes for SCN1A gene, 19 (68\%) entities out of total 28 were found to be directly associated with sodium channels. There were three $(50 \%)$ out of six chemicals with pharmacological effects that affect the functionality of sodium channels. The "disease concepts" are too broad to be linked directly to sodium channels. Rather these could be linked more appropriately with a specific disease and in the case of SCN1A gene, it is epilepsy.

\section{Future directions}

In future, as the list of the entities will grow and will be further curated, the improvements in the quality of dictionaries will certainly enhance the accuracy of the database. The comments obtained from the users will also help to incorporate changes that will make DDESC more useful. We also plan to include additional functionality like search options and batch query into the database in future revisions.

\section{Conclusion}

DDESC is aimed to serve as one-stop data warehouse for sodium channel research. The database could be of interest to researchers in different areas of biomedical and pharmaceutical research to identify targeted literature and possible associations/interactions between biological entities relevant to sodium channels. We hope that this database will serve as a useful complement to the existing 
public resources as being specialized for sodium channel biology. We also hope that the ability to generate association hypotheses could lead to new scientific concepts for further exploration. DDESC will be updated every six months and the information from all new studies published in that period will be incorporated.

\section{Abbreviations}

DDESC: Dragon Database for Exploration of Sodium Channels in Human; DES: Dragon Exploration System.

\section{Competing interests}

VBB and AR are partners in the OrionCell company whose product, Dragon Exploration System, has been used in creation of DDESC. Other authors declare no conflict of interest.

\section{Authors' contributions}

SS, MK and VBB conceptualized the study, analyzed results and wrote the manuscript. SS, MK, SVS, AD, AC and US performed the analysis. AR and VBB developed the DES system.

\section{Additional material}

\section{Additional file 1}

Comparison of genes and proteins results with other systems. Detailed comparison of the results for genes and proteins identified by PolySearch and DDESC along with the detailed list of 45 DDESC genes.

Click here for file

[http://www.biomedcentral.com/content/supplementary/14712164-9-622-S1.doc]

\section{Acknowledgements}

SS, AD, SVS, AC, are supported fully, and MK, US, AR, VBB partly by the DST/NRF Research Chair grant 6475I. MK, US, AR, VBB are supported partly by the National Bioinformatics Network grants. MK has been supported by the postdoctoral fellowship from the Claude Leon Foundation, South Africa. VBB was partly supported by National Research Foundation grants $(62302,61070)$.

\section{References}

I. Catterall WA: From ionic currents to molecular mechanisms: the structure and function of voltage-gated sodium channels. Neuron 2000, 26:13-25.

2. Bezzina C, Veldkamp MW, Berg MP van Den, Postma AV, Rook MB, Viersma JW, et al.: A single $\mathbf{N a ( + )}$ channel mutation causing both long-QT and Brugada syndromes. Circ Res 1999, 85: $1206-1213$.

3. Chen Q, Kirsch GE, Zhang D, Brugada R, Brugada J, Brugada P, et al.: Genetic basis and molecular mechanism for idiopathic ventricular fibrillation. Nature 1998, 392:293-296.

4. George AL Jr: Inherited disorders of voltage-gated sodium channels. J Clin Invest 2005, I I 5:1990-1999.

5. Miller TM, Dias da Silva MR, Miller HA, Kwiecinski H, Mendell JR, Tawil R, et al.: Correlating phenotype and genotype in the periodic paralyses. Neurology 2004, 63:1647-I655.

6. Wang Q, Shen J, Li Z, Timothy K, Vincent GM, Priori SG, et al.: Cardiac sodium channel mutations in patients with long $Q T$ syn- drome, an inherited cardiac arrhythmia. Hum Mol Genet 1995 , 4:1603-1607.

7. Weiss LA, Escayg A, Kearney JA, Trudeau M, MacDonald BT, Mori M, et al.: Sodium channels SCNIA, SCN2A and SCN3A in familial autism. Mol Psychiatry 2003, 8: I86-194.

8. Dib-Hajj SD, Cummins TR, Black JA, Waxman SG: From genes to pain: Na v I.7 and human pain disorders. Trends Neurosci 2007, 30:555-563.

9. Waxman SG: Channel, neuronal and clinical function in sodium channelopathies: from genotype to phenotype. Nat Neurosci 2007, 10:405-409.

10. Catterall WA, Goldin AL, Waxman SG: International Union of Pharmacology. $X X X I X$. Compendium of voltage-gated ion channels: sodium channels. Pharmacol Rev 2003, 55:575-578.

II. Chou KC: Structural bioinformatics and its impact to biomedical science. Curr Med Chem 2004, I I :2 I 05-2 I 34.

12. Chou KC: Insights from modeling three-dimensional structures of the human potassium and sodium channels. J Proteome Res 2004, 3:856-86I.

13. Chou KC: Modelling extracellular domains of GABA-A receptors: subtypes I, 2, 3, and 5. Biochem Biophys Res Commun 2004, 3 1 6:636-642.

14. Schnell JR, Chou J]: Structure and mechanism of the $\mathbf{M} 2$ proton channel of influenza A virus. Nature 2008, $45 \mathrm{I}: 59 \mathrm{I}-595$.

15. Chou KC, Wei DQ, Zhong WZ: Binding mechanism of coronavirus main proteinase with ligands and its implication to drug design against SARS. Biochem Biophys Res Commun 2003, 308:|48-|5|.

16. Gao WN, Wei DQ, Li Y, Gao H, Xu WR, Li AX, et al:: Agaritine and its derivatives are potential inhibitors against HIV proteases. Med Chem 2007, 3:221-226.

17. Li Y, Wei DQ, Gao WN, Gao H, Liu BN, Huang CJ, et al:: Computational approach to drug design for oxazolidinones as antibacterial agents. Med Chem 2007, 3:576-582.

18. Wang JF, Wei DQ, Chen C, Li Y, Chou KC: Molecular modeling of two CYP2CI9 SNPs and its implications for personalized drug design. Protein Pept Lett 2008, I5:27-32.

19. Zhang R, Wei DQ, Du QS, Chou KC: Molecular modeling studies of peptide drug candidates against SARS. Med Chem 2006, 2:309-314.

20. Zheng H, Wei DQ, Zhang R, Wang C, Wei H, Chou KC: Screening for new agonists against Alzheimer's disease. Med Chem 2007, 3:488-493.

21. Sirois S, Wei DQ, Du Q, Chou KC: Virtual screening for SARSCoV protease based on KZ7088 pharmacophore points. J Chem Inf Comput Sci 2004, 44: I I I I-I I 22.

22. Dea-Ayuela MA, Perez-Castillo Y, Meneses-Marcel A, Ubeira FM, Bolas-Fernandez F, Chou KC, et al.: HP-Lattice QSAR for dynein proteins: experimental proteomics (2D-electrophoresis, mass spectrometry) and theoretic study of a Leishmania infantum sequence. Bioorg Med Chem 2008, 16:7770-7776.

23. Du Q, Mezey PG, Chou KC: Heuristic molecular lipophilicity potential (HMLP): a 2D-QSAR study to LADH of molecular family pyrazole and derivatives. J Comput Chem 2005, 26:46I-470.

24. Du QS, Huang RB, Chou KC: Recent advances in QSAR and their applications in predicting the activities of chemical molecules, peptides and proteins for drug design. Curr Protein Pept Sci 2008, 9:248-260.

25. Du QS, Huang RB, Wei YT, Du LQ, Chou KC: Multiple field three dimensional quantitative structure-activity relationship (MF-3D-QSAR). J Comput Chem 2008, 29:2II-2I9.

26. Gonzalez-Diaz H, Gonzalez-Diaz Y, Santana L, Ubeira FM, Uriarte E: Proteomics, networks and connectivity indices. Proteomics 2008, 8:750-778.

27. Prado-Prado FJ, Gonzalez-Diaz H, de la Vega OM, Ubeira FM, Chou KC: Unified QSAR approach to antimicrobials. Part 3: first multi-tasking QSAR model for input-coded prediction, structural back-projection, and complex networks clustering of antiprotozoal compounds. Bioorg Med Chem 2008, | 6:587|-5880.

28. Chou KC, Shen HB: Recent progress in protein subcellular location prediction. Anal Biochem 2007, 370: I- 16.

29. Chou KC, Shen HB: Cell-PLoc: a package of Web servers for predicting subcellular localization of proteins in various organisms. Nat Protoc 2008, 3:153-162. 
30. Chou KC, Shen HB: MemType-2L: a web server for predicting membrane proteins and their types by incorporating evolution information through Pse-PSSM. Biochem Biophys Res Commun 2007, 360:339-345.

31. Shen HB, Chou KC: EzyPred: a top-down approach for predicting enzyme functional classes and subclasses. Biochem Biophys Res Commun 2007, 364:53-59.

32. Chou KC, Shen HB: Protldent: a web server for identifying proteases and their types by fusing functional domain and sequential evolution information. Biochem Biophys Res Commun 2008, 376:321-325.

33. Chou KC: A vectorized sequence-coupling model for predicting HIV protease cleavage sites in proteins. J Biol Chem 1993, 268: 16938-16948.

34. Chou KC: Prediction of human immunodeficiency virus protease cleavage sites in proteins. Anal Biochem 1996, 233:1-14.

35. Shen HB, Chou KC: HIVcleave: a web-server for predicting human immunodeficiency virus protease cleavage sites in proteins. Anal Biochem 2008, 375:388-390.

36. Chou KC, Shen HB: Signal-CF: a subsite-coupled and windowfusing approach for predicting signal peptides. Biochem Biophys Res Commun 2007, 357:633-640.

37. Shen HB, Chou KC: Signal-3L: A 3-layer approach for predicting signal peptides. Biochem Biophys Res Commun 2007, 363:297-303.

38. Pan H, Zuo L, Choudhary V, Zhang Z, Leow SH, Chong FT, et al.: Dragon TF Association Miner: a system for exploring transcription factor associations through text-mining. Nucleic Acids Res 2004, 32:W230-W234.

39. Bajic VB, Veronika M, Veladandi PS, Meka A, Heng MW, Rajaraman K, et al.: Dragon Plant Biology Explorer. A text-mining tool for integrating associations between genetic and biochemical entities with genome annotation and biochemical terms lists. Plant Physiol 2005, I 38:19|4-1925.

40. Kaur M, Radovanovic A, Essack M, Schaefer U, Maqungo M, Kibler T, et al.: Database for exploration of functional context of genes implicated in ovarian cancer. Nucleic Acids Res 2008.

4I. Malik R, Franke L, Siebes A: Combination of text-mining algorithms increases the performance. Bioinformatics 2006, 22:2I5I-2I57.

42. Mulley JC, Scheffer IE, Petrou S, Dibbens LM, Berkovic SF, Harkin LA: SCNIA mutations and epilepsy. Hum Mutat 2005, 25:535-542.

43. Cheng D, Knox C, Young N, Stothard P, Damaraju S, Wishart DS: PolySearch: a web-based text mining system for extracting relationships between human diseases, genes, mutations, drugs and metabolites. Nucleic Acids Res 2008, 36:W399-W405.

Publish with Bio Med Central and every scientist can read your work free of charge

"BioMed Central will be the most significant development for disseminating the results of biomedical research in our lifetime. "

Sir Paul Nurse, Cancer Research UK

Your research papers will be:

- available free of charge to the entire biomedical community

- peer reviewed and published immediately upon acceptance

- cited in PubMed and archived on PubMed Central

- yours - you keep the copyright
BioMedcentral 